\section{Compressed channel estimation for massive MIMO-OFDM systems over doubly selective channels}

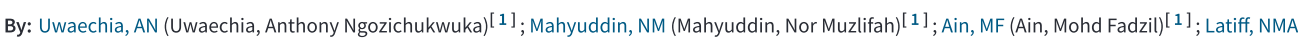
(Latiff, Nurul Muazzah Abdul) ${ }^{[2]}$; Za'bah, NF (Za'bah, Nor Farahidah) ${ }^{[3]}$

View Web of Science ResearcherID and ORCID

PHYSICAL COMMUNICATION

Volume: 36

Article Number: UNSP 100771

DOI: $10.1016 /$ j.phycom.2019.100771

Published: OCT 2019

Document Type: Article

View Journal Impact

\section{Abstract}

Doubly selective (DS) channel estimation for the downlink massive multiple-input-multiple-output orthogonal frequency division multiplexing (MIMOOFDM) systems is a challenging problem, due to the requirements on high pilots overhead and prohibitive complexity. In this paper, by exploiting the highly correlated spatial structure of the obtained array response vectors and sparsity of the multipath signal components of the massive MIMO-OFDM channels, a modified spatial basis expansion model (modified-SBEM) is introduced. Thus, using complex exponential (CE-) modified-SBEM (i.e., modified CE-SBEM) can improve the resolution of the angles of departures (AoDs) information to represent the downlink with far fewer parameter dimensions, since the AoDs are much slower than path gains. Subsequently, we jointly design the effective pilot power and pilot placement for sparse channel estimation by means of an extended model. Our design is based on the block-coherence and sub-coherence simultaneous minimization of the measurement matrix associated with the massive MIMO-OFDM system pilot subcarriers. Furthermore, we leverage the sparse nature of the massive MIMOOFDM system to formulate the quantized AoDs estimation into a block-sparse signal recovery problem, where the measurement matrix is designed based on the estimated virtual AoD. Thus, a new algorithm namely, generalized quasi-block simultaneous orthogonal matching pursuit (gQBSO), is introduced to solve the problem by providing sparse signal reconstruction solution. Simulation results demonstrate that the proposed scheme can effectively estimate the DS channel for massive MIMOOFDM systems compared with other existing algorithms. For example, at $\mathrm{SNR}=20 \mathrm{~dB}$ for $\mathrm{K}=4$ users, Doppler shift $=0.093$ with N-T $=32$ antenna size, the adaptive-QBSO algorithm with G-SBEM and the proposed gQBSO with modified-SBEM can realize approximately $75.44 \%$ and $85.14 \%$ of the NMSE achieved by the oracle estimator with modified-SBEM. (C) 2019 Elsevier B.V. All rights reserved.

\section{Keywords}

Author Keywords: Spatial basis expansion model (SBEM); Communication channels; Compressed sensing (CS); Channel estimation; Doubly selective (DS) channel; Sparse matrices; OFDM; Massive MIMO; Pilot design

KeyWords Plus: SYMBOL DESIGN; TRANSMISSIONS; EQUALIZATION; CAPACITY

\section{Author Information}

Reprint Address: Mahyuddin, NM (reprint author)

+ Univ Sains Malaysia, Sch Elect \& Elect Engn, Nibong Tebal 14300, Malaysia.

\section{Addresses:}

+ [1] Univ Sains Malaysia, Sch Elect \& Elect Engn, Nibong Tebal 14300, Malaysia

+ [2] Univ Teknol Malaysia, Sch Elect Engn, Utm Johor Bahru 81310, Johor, Malaysia

+ [3] Int Islamic Univ Malaysia, Dept Elect \& Comp Engn, Kuala Lumpur 53100, Malaysia

E-mail Addresses: eemnmuzlifah@usm.my

Funding

\begin{tabular}{|c|c|c|}
\hline Funding Agency & Show details & Grant Number \\
\hline \multicolumn{3}{|c|}{ School of Electrical and Electronic Engineering, Universiti Sains Malaysia, Malaysia } \\
\hline Ministry of Education, Malaysia & & FRGS 203/PELECT/6071373 \\
\hline
\end{tabular}

View funding text

Publisher

ELSEVIER, RADARWEG 29, 1043 NX AMSTERDAM, NETHERLANDS

\section{Journal Information}

Impact Factor: Journal Citation Reports

\section{Categories / Classification}

Research Areas: Engineering; Telecommunications

Web of Science Categories: Engineering, Electrical \& Electronic; Telecommunications

\section{Citation Network}

In Web of Science Core Collection

0

Times Cited

Create Citation Alert

\section{2}

Cited References

View Related Records

Use in Web of Science

Web of Science Usage Count

\section{4}

Last 180 Days

4

Learn more

This record is from:

Web of Science Core Collection

- Science Citation Index Expanded

Suggest a correction

If you would like to improve the quality of the data in this record, please suggest a correction. 


\section{Cited References: 52}

1. Joint Spatial Division and Multiplexing-The Large-Scale Array Regime

Times Cited: 581

By: Adhikary, Ansuman; Nam, Junyoung; Ahn, Jae-Young; et al.

IEEE TRANSACTIONS ON INFORMATION THEORY Volume: 59 Issue: 10 Pages: 6441-6463 Published: OCT 2013

2. Multisignal Compressed Sensing for Polarimetric SAR Tomography

Times Cited: 33

By: Aguilera, Esteban; Nannini, Matteo; Reigber, Andreas

IEEE GEOSCIENCE AND REMOTE SENSING LETTERS Volume: 9 Issue: 5 Pages: 871-875 Published: SEP 2012

3. Compressed Channel Sensing: A New Approach to Estimating Sparse Multipath Channels

Times Cited: 575

By: Bajwa, Waheed U.; Haupt, Jarvis; Sayeed, Akbar M.; et al.

PROCEEDINGS OF THE IEEE Volume: 98 Issue: 6 Pages: 1058-1076 Published: JUN 2010

4. Pilot Decontamination in Wideband Massive MIMO Systems by Exploiting Channel Sparsity

Times Cited: 31

By: Chen, Zhilin; Yang, Chenyang

IEEE TRANSACTIONS ON WIRELESS COMMUNICATIONS Volume: 15 Issue: 7 Pages: 5087-5100 Published: JUL 2016

5. Channel Estimation for OFDM Systems over Doubly Selective Channels: A Distributed Compressive Sensing Based Approach

Times Cited: 69

By: Cheng, Peng; Chen, Zhuo; Rui, Yun; et al.

IEEE TRANSACTIONS ON COMMUNICATIONS Volume: 61 Issue: 10 Pages: 4173-4185 Published: OCT 2013

6. FDD Massive MIMO Channel Estimation With Arbitrary 2D-Array Geometry

Times Cited: 29

By: Dai, Jisheng; Liu, An; Lau, Vincent K. N.

IEEE TRANSACTIONS ON SIGNAL PROCESSING Volume: 66 Issue: 10 Pages: 2584-2599 Published: MAY 152018

7. Subspace Pursuit for Compressive Sensing Signal Reconstruction

Times Cited: 1,219

By: Dai, Wei; Milenkovic, Olgica

IEEE TRANSACTIONS ON INFORMATION THEORY Volume: 55 Issue: 5 Pages:2230-2249 Published: MAY 2009

8. Title: [not available]

Times Cited: 1

By: Davenport, M.; Duarte, M.; Eldar, Y.; et al.

Introduction to Compressed Sensing, in Compressed Sensing: Theory and Applications Pages: 1-68 Published: 2011

Publisher: Cambridge Univ. Press, Cambridge, U. K

[Show additional data]

9. Channel Estimation for Massive MIMO TDD Systems Assuming Pilot Contamination and Frequency Selective Fading

Times Cited: 7

By: de Figueiredo, Felipe A. P.; Cardoso, Fabbryccio A. C. M.; Moerman, Ingrid; et al.

IEEE ACCESS Volume: 5 Pages: 17733-17741 Published: 2017

10. Sparse and Redundant Representations From Theory to Applications in Signal and Image Processing Prologue

Times Cited: 1,532

By: Elad, Michael

SPARSE AND REDUNDANT REPRESENTATIONS: FROM THEORY TO APPLICATIONS IN SIGNAL AND IMAGE PROCESSING Pages: 3-15 Published: 2010

11. Title: [not available]

By: Eldar, Y. C.; Kutyniok, G.

Times Cited: 916

Compressed sensing: Theory and applications Published: 2012

Publisher: Cambridge University Press

12. Block-Sparse Signals: Uncertainty Relations and Efficient Recovery

Times Cited: 681

By: Eldar, Yonina C.; Kuppinger, Patrick; Boelcskei, Helmut

IEEE TRANSACTIONS ON SIGNAL PROCESSING Volume: 58 Issue: 6 Pages: 3042-3054 Published: JUN 2010

13. Basis expansion models and diversity techniques for blind identification and equalization of time-varying channels

Times Cited: 398

By: Giannakis, GB; Tepedelenlioglu, $C$

PROCEEDINGS OF THE IEEE Volume: 86 Issue: 10 Pages: 1969-1986 Published: OCT 1998

14. Low Complexity ICI Mitigation for MIMO-OFDM in Time-Varying Channels

Times Cited: 4

By: Hao, Jinxing; Wang, Jintao; Pan, Changyong

IEEE TRANSACTIONS ON BROADCASTING Volume: 62 Issue: 3 Pages: 727-735 Published: SEP 2016

15. Pilot Allocation for Distributed-Compressed-Sensing-Based Sparse Channel Estimation in MIMO-OFDM Systems

By: He, Xueyun; Song, Rongfang; Zhu, Wei-Ping 
By: Hu, Zeng; Lin, Shaoe; Zheng, Beixiong; et al.

IEEE ACCESS Volume: 5 Pages: 23822-23832 Published: 2017

17. A Novel 3-D Massive MIMO Channel Model for Vehicle-to-Vehicle Communication Environments

Times Cited: 28

By: Jiang, Hao; Zhang, Zaichen; Dang, Jian; et al.

IEEE TRANSACTIONS ON COMMUNICATIONS Volume: 66 Issue: 1 Pages: 79-90 Published: JAN 2018

18. Joint Pilot Power \& Pattern Design for Compressive OFDM Channel Estimation

Times Cited: 12

By: Khosravi, Mahdi; Mashhadi, Saeed

IEEE COMMUNICATIONS LETTERS Volume: 19 Issue: 1 Pages: 50-53 Published: JAN 2015

19. Massive MIMO for Next Generation Wireless Systems

Times Cited: 2,546

By: Larsson, Erik G.; Edfors, Ove; Tufvesson, Fredrik; et al.

IEEE COMMUNICATIONS MAgAZINE Volume: 52 Issue: 2 Pages: 186-195 Published: FEB 2014

20. Estimation of Sparse Massive MIMO-OFDM Channels With Approximately Common Support

Times Cited: 16

By: Lin, Xincong; Wu, Sheng; Kuang, Linling; et al.

IEEE COMMUNICATIONS LETTERS Volume: 21 Issue: 5 Pages: 1179-1182 Published: MAY 2017

21. An Overview of Massive MIMO: Benefits and Challenges

Times Cited: 993

By: Lu, Lu; Li, Geoffrey Ye; Swindlehurst, A. Lee; et al.

IEEE JOURNAL OF SELECTED TOPICS IN SIGNAL PROCESSING Volume: 8 Issue: 5 Pages: 742-758 Published: OCT 2014

22. Title: [not available]

Times Cited: 17

Group Author(s): M. ApS

The MOSEK Optimization Toolbox for MATLAB Manual. vers. 7. 1 (rev. 28). Published: 2015

[Online]. Available

23. Maximum-diversity transmissions over doubly selective wireless channels

Times Cited: 147

By: Ma, XL; Giannakis, GB

IEEE TRANSACTIONS ON INFORMATION THEORY Volume: 49 Issue: 7 Pages: 1832-1840 Published: JUL 2003

24. Optimal training for block transmissions over doubly selective wireless fading channels

Times Cited: 300

By: Ma, XL; Giannakis, BB; Ohno, S

IEEE TRANSACTIONS ON SIGNAL PROCESSING Volume: 51 Issue: 5 Pages: 1351-1366 Published: MAY 2003

25. MIMO-OFDM Pilot Symbol Design For Sparse Channel Estimation

Times Cited: 3

By: Mohammadian, Roozbeh; Amini, Arash; Khalaj, Babak H.; et al.

2015 ASIA-PACIFIC SIGNAL AND INFORMATION PROCESSING ASSOCIATION ANNUAL SUMMIT AND CONFERENCE (APSIPA) Book Series: Asia-Pacific Signal and Information Processing Association Annual Summit and Conference Pages: 975-980 Published: 2015

26. Compressive Sensing-Based Pilot Design for Sparse Channel Estimation in OFDM Systems

Times Cited: 18

By: Mohammadian, Roozbeh; Amini, Arash; Khalaj, Babak Hossein

IEEE COMmUNICATIONS LETTERS Volume: 21 Issue: 1 Pages: 4-7 Published: JAN 2017

27. Signal Recovery From Incomplete and Inaccurate Measurements Via Regularized Orthogonal Matching Pursuit

Times Cited: 404

By: Needell, Deanna; Vershynin, Roman

IEEE JOURNAL OF SELECTED TOPICS IN SIGNAL PROCESSING Volume: 4 Issue: 2 Pages: 310-316 Published: APR 2010

28. Preamble and pilot symbol design for channel estimation in OFDM systems with null subcarriers

By: Ohno, Shuichi; Manasseh, Emmanuel; Nakamoto, Masayoshi

EURASIP JOURNAL ON WIRELESS COMMUNICATIONS AND NETWORKING Article Number: 2 Published: 2011

29. Joint Design of Pilot Power and Pilot Pattern for Sparse Cognitive Radio Systems

By: Qi, Chenhao; Wu, Lenan; Huang, Yongming; et al.

IEEE TRANSACTIONS ON VEHICULAR TECHNOLOGY Volume: 64 Issue: 11 Pages: 5384-+ Published: NOV 2015

30. Sparse Channel Estimation for Massive MIMO-OFDM Systems Over Time-Varying Channels

Times Cited: 8

By: Qin, Qibo; Gui, Lin; Gong, Bo; et al.

IEEE ACCESS Volume: 6 Pages: 33740-33751 Published: 2018

Showing $\mathbf{3 0}$ of $\mathbf{5 2}$ View All in Cited References page 
